\title{
Uncertainty quantification and sensitivity studies on Thorium-fueled reactors
}

\author{
Eliot Party ${ }^{1, *}$, Xavier Doligez ${ }^{2}$, Philippe Dessagne ${ }^{1}$, Maëlle Kerveno ${ }^{1}$, and Greg Henning ${ }^{1}$ \\ ${ }^{1}$ Université de Strasbourg, CNRS, IPHC UMR 7178, 23 Rue du Loess BP28 67037 Strasbourg \\ ${ }^{2}$ Université Paris 11, IN2P3-CNRS, IPNO, 15 rue Georges Clemenceau, 91406 Orsay, France
}

\begin{abstract}
This paper shows how Total Monte Carlo (TMC) method and Perturbation Theory (PT) can be applied to quantify uncertainty due to nuclear data on reactor static calculations of integral parameters such as $k_{\text {eff }}$ and $\beta_{\text {eff }}$. This work focuses on thorium fueled reactors and it aims to rank different cross sections uncertainty regarding criticality calculations. The consistency of the two methods are first studied. The cross sections set used for the TMC method is computed to build adequate correlation matrices. Those matrices are then multiplied by the sensitivity coefficients obtained thanks to the PT to obtain global uncertainties that are compared to the ones calculated by the TMC method. Results in good agreement allow us to use correlation matrix from the state of the art nuclear data library (JEFF 3-3) that provide insight of uncertainty on $k_{e f f}$ and $\beta_{e f f}$ for thorium fueled Pressurized Water Reactors. Finally, maximum uncertainties on cross sections are estimated to reach a target uncertainty on integral parameters. It is shown that a strong reduction of the current uncertainty is needed and consequently, new measurements and evaluations have to be performed.
\end{abstract}

\section{Introduction}

Studies of innovative nuclear systems rely on precise computational tools and reliable nuclear data. Accordingly, among other sources of uncertainties, cross sections are today of prime importance for criticality calculations, and specifically for $k_{e f f}$ and $\beta_{e f f}$ estimations. For several years some efforts have been made to propagate nuclear data uncertainty on reactor's calculations. To this end, two main methods have been widely developed and used : the Total Monte Carlo (TMC) [1] method and the Perturbation Theory (PT) [2]. They have been applied to current and innovative reactors [3] to estimate integral parameters uncertainties, but also to justify some new experimental programs for nuclear data measurements.

Among innovative nuclear systems, the thorium fuel cycle option is less studied than the uranium/plutonium cycle. Unsurprinsingly, related nuclear data show some strong uncertainties. Even if possible deployments of thorium fuel reactors is probably delayed to the second half of century, effort on nuclear data knowledge and specially new precise measurements with reliable uncertainties should be carried now as in [4]. Indeed, the time laps between a new measurement and an improvement on nuclear data libraries can reach at least one decade. This paper aims to quantify the uncertainty limits on cross sections to reach a precision smaller than $0.5 \%$ for $k_{e f f}$ and $5 \%$ for $\beta_{\text {eff }}$ as specified in [5].

The first part of the paper presents the tools and methods of this work. There, the TMC method and PT are detailed as well as the set of evaluations coming from TENDL-2013 [6] and the construction method of cross

*e-mail: eliot.party@etu.unistra.fr sections covariances derived from this set. Then, in a second part, results combining covariances from TENDL2013 and sensitivity coefficients calculated with PT are compared with TMC calculations and discussed. Finally, in the last part we present the estimation of the minimum uncertainties of cross sections to reach the target precision on $k_{e f f}$ and $\beta_{e f f}$ for thorium fuel PWR.

\section{Tools and methods}

All calculations performed for this work were done with the stochastic code SERPENT2 [7]. It has the ability to compute sensibility coefficients of several integral observable to basic parameters such as cross sections since several years [8]. The stochastic approach was chosen not to bring approximation due to self-shielding treatments for criticality calculations. However, all results are associated to a statistical uncertainty. For all simulations, we insured that this statistical uncertainty is effectively negligible in regards of those induced by nuclear data.

\subsection{Systems presentation and case study}

To test TMC and PT methodologies, we first consider a typical PWR assembly configuration, shown in Figure 1. It is a fully reflective geometry with reflective boundary conditions. The ${ }^{233} U$ enrichment is $3 \%$ to be representative of a beginning of cycle composition. No boron acid neither control roads are considered on the simulations: guide tubes and the instrumentation tube are filled with water. 


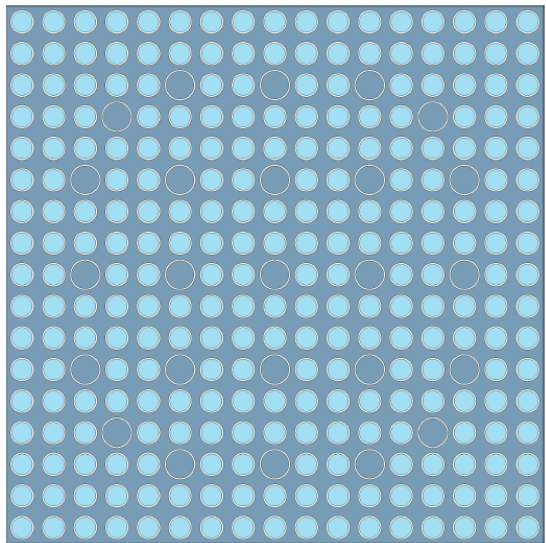

Figure 1. (color online) PWR Assembly geometry. Zirconium sheath (white), $\mathrm{TH} / \mathrm{UO}_{2}$ fuel (blue), light water (grey).

\subsection{Uncertainties and sensibility calculations}

\subsubsection{Uncertainties with the TMC method}

The TMC method is now currently used for global uncertainties calculations. The main idea is to sample a great number of different nuclear parameters to build a set of cross section evaluations, whose dispersion is representative of the uncertainty of the cross sections. As an example, Figure 2 presents 7 different evaluations of the ${ }^{233} U$ fission reaction.

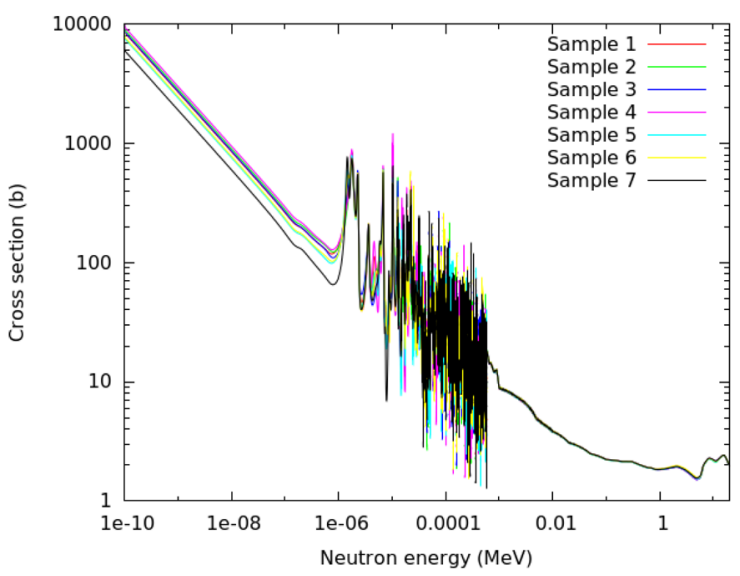

Figure 2. (color online) Extraction of different evaluations for ${ }^{233} U(n, f)$ reaction taken from the TENDL-2013 library.

As each nuclear parameter impacts all reaction cross sections, it is not possible to isolate the contribution of a single reaction with this method. The main advantage relies in the exact estimation in cross sections. The main drawback is the computational cost: for each evaluation considered in the sample, a calculation is needed. For this work, a set of 300 evaluations for both ${ }^{232} \mathrm{Th}$ and ${ }^{233} \mathrm{U}$ is used, leading to a total of 600 different calculations. No cross sampling were considered here, assuming that thorium and uranium evaluations are completely independent.
Table 1. $k_{\text {eff }}$ uncertainties due to ${ }^{233} \mathrm{U}$ and ${ }^{232} \mathrm{Th}$ calculated with the TMC method.

\begin{tabular}{cc}
\hline Observable & $k_{\text {eff }}$ Uncertainty \\
\hline${ }^{233} U$ & $5.82 \%$ \\
${ }^{232} \mathrm{Th}$ & $1.06 \%$
\end{tabular}

Each calculation lasts 8 h.cpu (to reach a negligible statistical uncertainty) leading to a complete computational time of 4800 h.cpu.

The set of evaluations used here is from TENDL2013 [6]. $k_{\text {eff }}$ calculations distribution for the PWR assembly is presented in Figure 3 as an example.

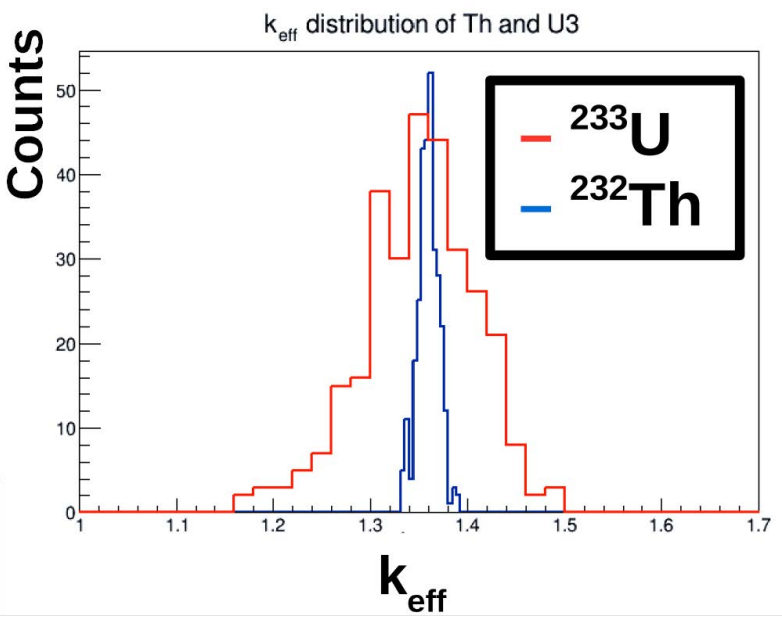

Figure 3. (color online) $k_{\text {eff }}$ distribution obtained for PWR assembly calculations, varying the ${ }^{233} \mathrm{U}$ evaluation in red and the ${ }^{232} \mathrm{Th}$ in blue.

From those types of distributions, the standard deviation is extracted and then the uncertainties due to ${ }^{232} \mathrm{Th}$ and ${ }^{233} U$ related nuclear data are deduced. Results obtained for the PWR configuration are presented in table 1.

\subsubsection{Covariance matrix and perturbation theory}

Another way to use the set of evaluations from TENDL2013 , is to calculate cross sections correlations. The energy domain is binned within a given energy group structure. The covariance matrix, is then obtained by the statistical estimations of the correlation between each term of the matrix as in expression 1.

$$
\operatorname{cov}\left(\sigma_{i}^{g}, \sigma_{j}^{g^{\prime}}\right)=\frac{E\left(\sigma_{i}^{g} \sigma_{j}^{g^{\prime}}\right)-E\left(\sigma_{i}^{g}\right) E\left(\sigma_{j}^{g^{\prime}}\right)}{\sqrt{\operatorname{var}\left(\sigma_{i}^{g}\right)} \cdot \sqrt{\operatorname{var}\left(\sigma_{j}^{g^{\prime}}\right)}}
$$

where $\sigma_{i}^{g}$ stands for one cross section reaction (quoted $i$ of the group $g, \sigma_{j}^{g^{\prime}}$ represents another reaction and another energy group, $E\left(\sigma_{i}^{g}\right)$ stands for the mathematical expectation of the given cross section reaction and $\operatorname{var}\left(\sigma_{j}^{g}\right)$ is the variance of this reaction cross section. Calculating expression 1 for all reactions and all energy group, it is 
possible to build covariance matrix from the set of evaluations given by TENDL-2013. As an example, the matrix for ${ }^{233} U(n, f)$ reaction is plotted in Figure 4.

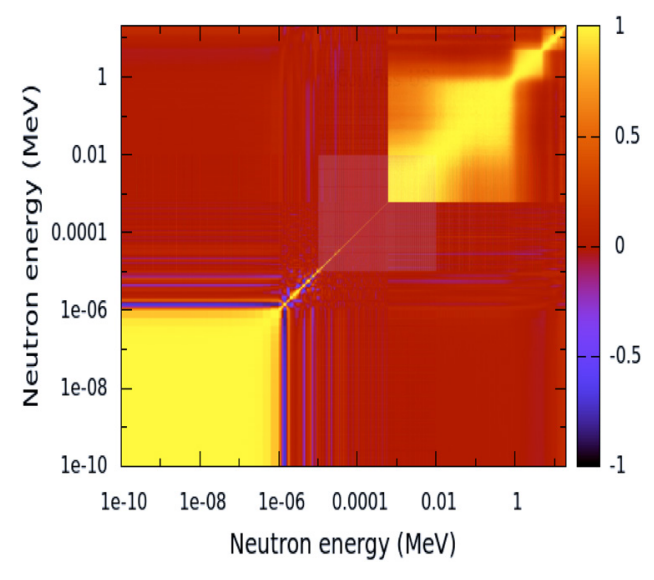

Figure 4. (color online) ${ }^{233} \mathrm{U}(\mathrm{n}, \mathrm{f})$ covariance matrix (for different energy bins).

Sensitivity coefficients are computed within the same energy group structure with SERPENT2. In function of neutron energy, an example of a sensitivity coefficient to $k_{e f f}$ is plotted in Figure 5. With those sensitivity coefficients, and the corresponding covariance matrix, it is possible to estimate uncertainties of integral parameters from expression 2

$$
\operatorname{var}\left(k_{e f f}\right)=S_{k}^{T}(\sigma) \cdot \operatorname{Cov}(\sigma, \sigma) \cdot S_{k}(\sigma)
$$

where $S_{k}(\sigma)$ represents the sensitivity coefficients vector and $\operatorname{Cov}(\sigma, \sigma)$ is the covariance matrix calculated thanks to expression 1.

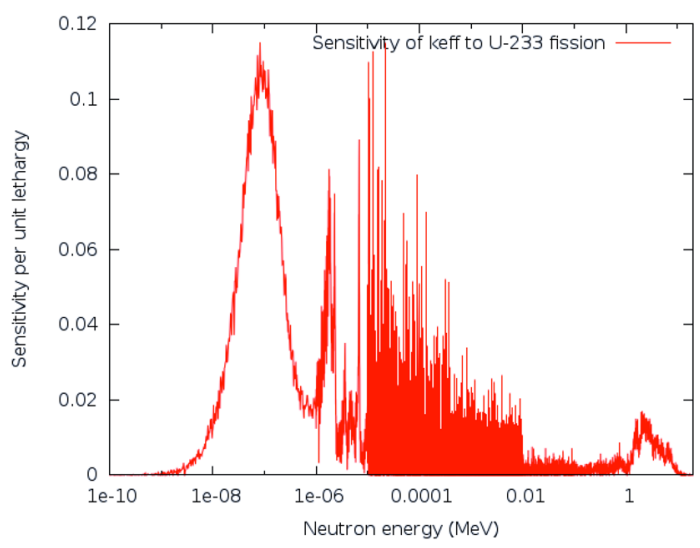

Figure 5. (color online) $k_{\text {eff }}$ sensibility to ${ }^{233} \mathrm{U}(\mathrm{n}, \mathrm{f})$ reaction.

The influence of the group structure has been studied as it is a parameter of first importance on the covariance calculation. We observed good agreement between TMC calculations and PT methods when the number of energy bin is high enough as it can be seen in Figure 6. This plot
Table 2. Typical $k_{\text {eff }}$ and $\beta_{\text {eff }}$ uncertainties for fast and thermal neutron spectrum due to ${ }^{233} \mathrm{U}$ and ${ }^{232} \mathrm{Th}$ cross sections.

\begin{tabular}{ccc}
\hline Observable & thermal spectrum & fast spectrum \\
\hline$k_{\text {eff }}$ & $0.49 \%$ & $1.63 \%$ \\
$\beta_{\text {eff }}$ & $0.23 \%$ & $1.98 \%$ \\
\hline
\end{tabular}

shows the uncertainty calculation with the PT method for a different number of energy groups (in abscissa). Value given by TMC calculations is reminded and is considered as the reference value.

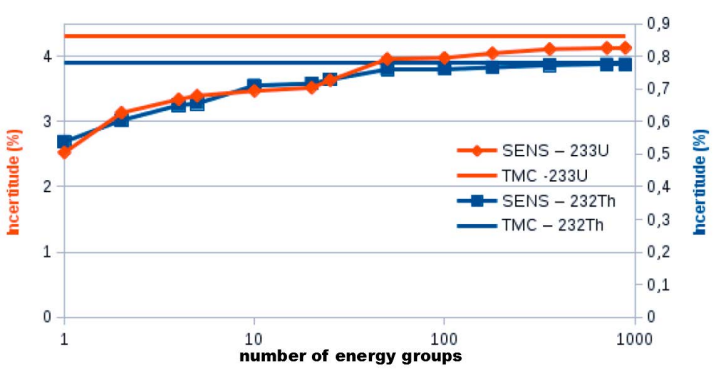

Figure 6. (color online) Uncertainty calculations with PT method regarding the energy group structure compared to TMC calculations.

As it can be seen, as soon as the number of groups is high enough (greater than approximately 100 for this work), PT is in good agreement with TMC method. It has the advantage to be cheeper in terms of computational effort. Indeed, one single calculation that gives sensitivity coefficient associated to covariance matrix lasts approximately 58 h.cpu, much less than the computational time needed for the TMC method.

\section{Results and discussion}

For the following, an energy structure of 1000 bins have been chosen in order to estimate uncertainty with the state of the art covariance matrix taken from the JEFF 3.3 library for $\beta_{\text {eff }}$ and $k_{\text {eff }}$ calculations. Two different system geometries have been used for this estimation. The first one is the PWR assembly considered in the previous section. The second one is a bare sphere made of ${ }^{232} \mathrm{Th}$ and ${ }^{233} \mathrm{U}$ mix oxide. The enrichment have been calculated to reach criticality. This very simple geometry is used to as an academic example of a fast neutron spectrum.

Application of the PT gives results that are presented in the table 2. It has to be pointed out that only cross section uncertainties have been propagated. Neither $v$, the number of neutron emitted by fission, neither the delayed neutron fraction have been considered as sources of uncertainties

The thermal configuration reaches the target precision both for $k_{e f f}$ and $\beta_{e f f}$ but the precision of $k_{e f f}$ calculations for the sphere is way out. The PT method allows us to estimate the maximal uncertainties cross section should have to reach the target of $0.5 \%$ uncertainty on $k_{\text {eff }}$. Results are shown in table 3 , considering or not correlations 
Table 3. Current uncertainties (JEFF 3.3) and target uncertainties for main reactions affecting $k_{\text {eff }}$ accuracy in the relevant neutron range from $1 \mathrm{keV}$ to $10 \mathrm{MeV}$.

\begin{tabular}{cccc}
\hline & JEFF & $\begin{array}{c}\text { With } \\
\text { Correlation }\end{array}$ & $\begin{array}{c}\text { Without } \\
\text { Correlation }\end{array}$ \\
\hline${ }^{233} U(n, f)$ & $1 \%$ & $1 \%$ & $0.45 \%$ \\
${ }^{232} T h(n, \gamma)$ & $3 \%$ & $3 \%$ & $0.8 \%$ \\
${ }^{233} U(n, \gamma)$ & $15 \%$ & $5 \%$ & $5 \%$ \\
\hline
\end{tabular}

within those cross sections (and assuming that the correlations calculated with TENDL-2013 are valid). In this table, current cross section typical uncertainties from JEFF 3.3 library are reminded.

If ${ }^{232} \operatorname{Th}(n, \gamma)$ and ${ }^{233} U(n, f)$ uncertainties were strongly correlated (for example if they were measured precisely against the same standard), then moderate precision gain is necessary to achieve $0,5 \%$ target due to compensation effects between reactions. In case they are uncorrelated, stringent criteria are needed to achieve the same precision. Therefore, correlations between different isotopes and reactions are essential for uncertainty propagation. If no covariance are taken into account, a tremendous effort on evaluation precision have to be carried out to reach such low uncertainties for cross sections. Those uncertainty limits might be even smaller as only cross section uncertainties were considered in this work whereas other sources of uncertainties are present.

\section{Conclusion and perspectives}

This work presents sensitivity and uncertainties analysis for $k_{e f f}$ and $\beta_{\text {eff }}$ calculations in thorium cycle for two types of neutron spectrum: fast and thermal. Perturbation theory and Total Monte Carlo methods, two classical ways to assess uncertainty issues, are applied here and put into perspectives. On one hand, the TMC method allows direct calculations of uncertainties, it is very costly in terms of computational power and the results may be difficult to discuss as all nuclear parameters are changing from an evaluation to another. On the other hand, the PT is based on sensitivity calculations that underline the parameters (cross sections) of prime importance in criticality calculations (mock-ups are designed based on sensitivity calculations). Also the PT is cheap in terms of computational efforts, it does not allow direct uncertainty calculation as cross sections are correlated. The link between PT and TMC methods is then the covariance matrix which represents uncertainties for all cross sections and correlations between them. This paper shows how the TENDL libraries, here TENDL-2013, can be computed to produce those correlation matrix without any criticality calculations. Comparisons between PT and TMC methods for uncertainties are in very good agreement as soon as the energy group structure for sensitivity coefficient (and for correlation matrix) is fine enough. An optimization of the group structure could be performed but in this work, regarding the computational power saving due to the use of PT, we used a 1000 bins group structure for calculations. The set of evaluations used for ${ }^{232} \mathrm{Th}$ and ${ }^{233} U$ were not optimized either, that is why the uncertainties on $k_{e f f}$ shown in this paper are quite large. A Bayesian Monte Carlo Approach [9] that may introduce constraints thanks to integral experiments analysis would constraint the results and give much narrower uncertainties. The next step would be the computation of the covariance matrix with this Bayesian Monte Carlo Method but it would require extra criticality calculations and then would increase the computational cost of the PT with correlations computed with TENDL. Finaly, the covariance matrix from JEFF 3.3 was considered in this work to estimate target uncertainty for different cross sections in order to reach a $k_{\text {eff }}$ uncertainty below than $0.5 \%$ and a $\beta_{\text {eff }}$ uncertainty below $5 \%$ as requested in [9]. Results show that some great efforts have to be carried out on new precise measurements, specially for the ${ }^{233} U(n, \gamma)$ reaction.

\section{References}

[1] A.J. Koning, D. Rochman, "Modern Nuclear Data Evaluation with the TALYS Code System", Nuclear Data Sheets, Volume 113, Issue 12, 2012, p2841-2934

[2] G. Aliberti, G. Palmiotti, M. Salvatores, T.K. Kim, T.A. Taiwo, M. Anitescu, I. Kodeli, E. Sartori, J.C. Bosq, J. Tommasi, "Nuclear data sensitivity, uncertainty and target accuracy assessment for future nuclear systems", Annals of Nuclear Energy, Volume 33, Issue 8, 2006, p700-733

[3] D. Rochman, A.J. Koning, S.C. Van Der Mark, A. Hogenbirk, C.M. Sciolla, "Nuclear data uncertainty propagation : Perturbation vs Monte Carlo", Annals of Nuclear Energy, Volume 38, Issue 5, 2011, p942-952

[4] E. Party, C. Borcea, P. Dessagne, X. Doligez, G. Henning, M. Kerveno, A. Negret, M. Nyman, A. Olacel, A. Plompen, "Neutron inelastic scattering off 232Th: measurements and beyond", WONDER 2018 : 5th International Workshop On Nuclear Data Evaluation for Reactor applications, conference proceedings, Aix-enProvence, France, 2018.

[5] Argonne National Laboratory - Nuclear Engineering Division, "Requirements for Advanced Simulation of Nuclear Reactor and Chemical Separation Plants", Report number ANL-AFCI-168, 2006, p30

[6] A.J. Koning, D. Rochman, S.C van der Marck, J. Kopecky, J. Ch. Sublet, S. Pomp, H. Sjostrand, R. Forrest, E. Bauge, H. Henriksson, O. Cabellos, S. Goriely, J. Leppanen, H. Leeb, A. Plompen, R. Mills, "TENDL2013: TALYS-based evaluated nuclear data library", 2013

[7] J. Leppänen, "SERPENT Monte Carlo reactor physics code", 2010

[8] M. Aufiero et al., "A collision history-based approach to sensitivity/perturbation calculations in the continuous energy Monte Carlo code SERPENT', Annals of Nuclear Energy, Volume 85, 2015, p245-258

[9] A.J. Koning, "Bayesian Monte Carlo Method for Nuclear Data Evaluation", Nuclear Data Sheets, Volume 123, 2015, p207-213 